\title{
Chronillogicalities: Déjà vus and hallucinations in the digital semiosphere
}

\author{
${ }^{1}$ Shanghai University, Shanghai, China, E-mail: massimo.leone@unito.it \\ ${ }^{2}$ University of Turin, Turin, Italy, E-mail: massimo.leone@unito.it
}

\begin{abstract}
:
The semiotics of phenomena like déjà vus and hallucinations constitute a limit-field of a theory of the sign, but one that offers opportunities to question the fundamental principles of the discipline while at the same time offering the opportunity to address their underlying cognitive processes. The article describes the cognitive nature of déjà vus and hallucinations, briefly reviews the literature about them, and reads them as cognitive perturbations in the light of a semiotics of mental simulacra related to perception, apperception, awareness, memory, and imagination. The article then uses such cognitive and semiotic modeling in order to develop a critique of present-day digital culture, in which the uncritical adoption of a mnemonic ideal based on digital memory jeopardizes one of the key features of embodied memory: imperfection and, as a consequence, the possibility to access aesthetic and temporal singularity. A collective memory prone to déjà vus and hallucinations ensues.
\end{abstract}

Keywords: déjà vu, hallucination, temporal cognition, semiotics, collective memory

DOI: 10.1515/cogsem-2019-2012

Every sane person lives in a double world, the outer and the inner world, the world of percepts and the world of fancies [...] a man can be durably affected by his percepts and by his fancies. The way in which they affect him will be apt to depend upon his inborn disposition and upon his habits.

(Peirce 1907, CP 5.4: 87)

\section{The phenomenology of déjà vus: State of the art and semiotic problematization}

Déjà vus are psychological events consisting in the impression of recognizing a situation that is actually experienced for the first time. Literature on déjà vus is interdisciplinary but predominantly psychological. It includes Jansen (1991), a phenomenological inquiry; Sno (1993), a doctoral thesis with a psychiatric perspective; Brown (2004), an examination of existing psychological theories; Krapp (2004), which turns the temporal and psychological figure of the déjà vu into the cornerstone of the author's critique of the present-day rhetoric of memory; Bodei (2006), an interdisciplinary survey of previous insights and theories on the subject, within a philosophical framework; Houppermans et al. (2011), on the treatment of déjà vus by the arts; Blair (2012), including déjà vus in the semiology of epileptic episodes; Bruneau (2012), which deals with déjà vus in the line of Alfred Korzybski's key concept of 'Time-binding;' Strongman (2012), which retraces the history of scholarly interest in the phenomenon and, through qualitative analysis, proposes that déjà vu is a commonly occurring normal experience - an effect of temporary over-excitation of hippocampal synaptic transmission - with a purposeful cognitive function by acting as an orientation-reflex to spatial-temporal reflection in subjects' momentary consciousness; Zantides (2016), which investigates déjà vus in visual intertextuality; Pozzato (2017), which applies Benveniste's semiotics of enunciation on the analysis of a literary representation of historical déjà vu in Sebald; Jones and Flaxman (2019), a popularizing account of the phenomenon. Peirce focuses on déjà vus in MS 517: what happens in déjà $v u$ is that we have a feeling which is autonomous while we feel as if it is a resemblance to something antecedent; for a commentary, see Anderson (1984: 459).

Déjà vus are usually puzzling for they seem to illogically contradict the customary sequence of time. One has the feeling of having already heard a sentence, seen a room, or been in a situation, although the context suggests that this could not be possibly the case. Déjà vus are startling, first, because they hint at the possibility of subverting the order of time - as though it was suddenly able, albeit for a short period, to flow backward; second, they are disconcerting because, having for a moment the feeling of being able to remember the present, one is also led to believe, for a single moment as well, to be able to remember the future of such present. Indeed, 
one is encouraged to assume, albeit for an instant, that the déjà vu might be a sort of door to access one's future from the present, a present experienced as past remembered from a more remote future, which is suddenly drawn into the present itself and allows one to remember it as past and, possibly, as present leading to a future that is already past. That causes that psychological and pragmatic condition of short paralysis that usually characterizes the déjà vu: one stops acting into the world and becomes, for the time of the déjà vu, a spectator of one's life, observed not in medias res, as it is happening along the present, but from a vintage point situated into a future that casts its light onto the present.

The déjà vu is, nevertheless, embodied, since one is both the person who is experiencing the present and the person who is remembering it, hence the feeling of freezing, as though one was waiting for the flowing of time and events to confirm that what is being experienced for the first time had actually been already experienced and is, as a consequence, remembered. The hope to predict the future through a condition of déjà vu is, however, doomed to be frustrated: first, because that which is usually remembered in the present of a déjà vu is not a precise event - such as whether a football player will or will not score a penalty in a crucial game - but rather an atmosphere, which might nevertheless include complex cognitive contents, like fragments of conversation, for example. Someone tells something to us, and we have the concrete, compelling feeling that we have already heard that sentence. The impression of remembering, moreover, concerns not only and not as much the semantic content of the sentence, as the pragmatic conditions in which it is uttered: the lighting in the room where the sentence is pronounced, the tone of voice by which it is proffered, the precise instant of its manifestation, etc. As one concentrates on the content of the déjà vu and seeks to extract from it some knowledge concerning the possible future to follow, however, the déjà vu is usually dispelled, and that paradoxical superposition of present and future that has created it vanishes too, with one's cognition returning to the normality of a present that, unaware of the future, flows into it and simultaneously becomes the past of the future present: of the future that will be present, and of the present that will be in the future. Thus, the disquieting feeling of having experienced a temporary subversion of the laws of time, memory, and awareness also dwindles away, leaving one merely with the shadow of an unusual, slightly worrying event. Déjà vus last no more than few instants although they might be subjectively perceived as longer - and might be repetitively occur to an individual, but without ever losing their perceived nature of exceptionality.

\section{The cognitive physiology of déjà vus}

Déjà vus are explained with reference to a simple cognitive alteration (Jones and Flaxman 2019): in normal conditions, the part of the brain that retains information about the present, storing this information in the form of memory, works chronologically after the part of the brain that creates a feeling of experience and awareness of the present. In normal cognitive circumstances, thus, one sees a red tulip and then stores its image in one's memory, as well as the memory of having seen the red tulip, and possibly also the feeling of the intentional or spontaneous memorability of the red tulip, together with the series of semantic associations attached to it. In particular cognitive conditions, though, such as fatigue, for instance, this chronology of perception and memory, awareness and remembrance, is subverted: the brain stores an image of the red tulip, a memory of having seen the red tulip, and a feeling of its memorability before processing the perception of the red tulip, and also before giving rise to the feeling of the presence and awareness of such perception. As a consequence, when one eventually realizes that one is seeing a red tulip in the present and is aware of such experience, one already has in one's memory the stored simulacrum of both the red tulip, its perception, and the awareness of it. In a déjà vu, then, one is led to have the impression of recollecting one's awareness of something. That does not mean that awareness of perception systematically stems from memory, and this from the retention of perception. Several degrees of such retention can be posited in relation to the role of intentionality: at the one end of the spectrum, someone perceives something, such as the plaque of the car that was about to run them over, and makes an explicit and intentional effort to retain such image and its visual information; at the other end of the spectrum, a visual scenery is perceived but retained just for the instantaneous time that requires it to be perceived (perception would not be possible, indeed, without a certain time of retention of its content in one's memory, albeit only for a single instant): this is the case of the accidental witness to a car accident, who might perceive the scene, retain it in the memory, but without the explicit intentionality to transform it into a clear and persistent mnemonic content. Déjà vus are unlikely to arise when the intentionality of mnemonic retention is acute, whereas they are probably more common when retention takes place en passant and does not make the object of an explicit mnemonic effort. In such circumstances, when spontaneous retention gives shape to an unintentional memory that precedes the formation of a feeling of apperception, déjà vus arise as short-circuits in the chain of perception - mnemonic retention - memorization of perception - apperception - awareness of perception - awareness of memorization; the result is rather the chain of perception - mnemonic retention - 
apperception - memorization of perception - awareness of perception - awareness of memorization. It should be noticed that in this short-circuit, awareness of perception still follows memorization, for that should account for the fact that, in most déjà vus, the actual mnemonic content of the pseudo-memory is usually vague, and incomparable to that of an intentional memorization.

A déjà vu does not represent, however, a subversion of the ontology of time. In the ontology of the experience of a human body that comes into contact with a red tulip, for example, a mental simulacrum of the red tulip can be created only after that one has actually come across it. The human mind has an ontological dignity, the red tulip has it too, and the encounter between the former and the latter also remains in the ontological domain: there is, in reality, the ontological circumstance of an encounter between a human body, its mind, and a red tulip. The problem is that the mental simulacrum that allows a human body to experience a feeling of being in the presence of a red tulip, and of being aware of being in the presence of a red tulip, is, indeed, a simulacrum - that is, a mental representation whose phenomenological nature is not identical but similar to the phenomenological nature of the mental simulacrum that allows the same body to remember the experience of having seen a red tulip, and the awareness of such experience. When I see a red tulip, I do not see it in a pure instant, no matter how short the exposure of my sight to such red tulip might be, but in an instant that must have some kind of temporal continuity. It is an instant that is never a pure temporal point but rather a line that stretches throughout time, albeit for a brief time. Thus, when I see a red tulip, I can realize that I am seeing a red tulip because I am simultaneously remembering that I am seeing it while I am seeing it. I am not remembering it, however, because I have already seen it in the past, but because the mental simulacrum that allows me to have a present experience of the red tulip automatically turns into the mental simulacrum that allows me to have an ongoing awareness of being seeing a red tulip, and will also turn into the mental simulacrum that will allow me to remember to having been seeing a red tulip and to have had the awareness of seeing it.

\section{The semiotics of déjà vus}

As times goes by, then, the object of a perception becomes a semiotic one. That happens not only because the object inevitably works as the token of a type. When I see a red tulip, I recognize an object, and a living object, and a vegetable, and a flower, and a tulip, and a red tulip, because this articulation of experience is already deposited in my memory and shared with the language and culture that I have interiorized and that guides my segmentation of experience. Nevertheless, if I do not simply look at the tulip but I contemplate it, that is, if I concentrate on the idiosyncratic details that constitute the singularity of one specific tulip, then I must perceive the tulip not only as a token of several types but also as an object that turns into the source of a unique perception, some elements of which can, indeed, be categorized according to the specific token/type dialectics that is involved in the perception and cultural and linguistic categorization of such object, whereas some other
elements, on the contrary, exceed this dialectics and constitute a residue of it, giving rise to an encounter with singularity.

That is the typical experience of seeing a face for the first time, especially if, for mysterious reasons, such face appears as acutely and even irresistibly appealing to us, bringing about the persisting and compelling feeling of not being able to stop staring at such face, if not out of decency and social convenience. In such circumstances, we do of course perceive the face as the token of a type; we realize, for instance, that is a face, a human face, a female face, a Caucasian face, a young face, a pigmented face, with slanted eyes, slightly made up, etc. At the same time, we also perceive some features of the face that we cannot possibly categorize as tokens of types and that, as a result, constitute the uniqueness and singularity of that face. Up to a certain point, such face might resemble other faces that fall into similar categories; yet, the genetic variety that underpins the generation of human faces is such that we are doomed to perceive each face as endowed with at least some elements that cannot be categorized and that, as a consequence, compose the face's uniqueness.

Whenever singularity is encountered, then, it can only partially be perceived through comparison with mental simulacra of past experiences and with the models that are offered by the linguistic and cultural articulation of reality that one has interiorized. The encounter with singularity prompts the creation of a new mental simulacrum that must also sometimes radically change the pre-existent patterns of linguistic and cultural articulation of reality. Coming across a beautiful human face means having to restructure one's mental matrix according to which human faces are perceived and experienced. The perception of singularity is extremely relevant and interesting for semiotics exactly it because constitutes an advantageous case-study in order to analyze the way in which the process of a human body-mind complex, coming across an object in the ontology of reality, gives rise not only to perception, apperception, experience, formation of mental simulacra, awareness, and memory, but also to the semiotic conditions that are associated with these mental states and constitute their underpinning logic. 


\section{The recognition of the unseen}

When I see a beautiful face for the first time, the semiotic activity that this event triggers is very complex. The face must not necessarily be beautiful, but it is convenient to posit the example of a first encounter with a beautiful face because "beautiful" must work here as a shortcut adjective to indicate something that is singular - that is, that cannot be simply perceived through a token/type dialectics, and in the perception of which, moreover, such dialectics are largely insufficient to explain the nature of the mental activity that goes on when the beautiful face is come across. A caveat is necessary here, though. True beauty is likely to be singular, or at least to entail a high degree of singularity, meaning that it would be difficult to experience authentic aesthetic exceptionality in relation to something that can be easily categorized as the token of a type. At the same time, the opposite is not necessarily true: something might be considered singular, without for that reason immediately falling into the category of what is beautiful. Indeed, the monstrous too, to a certain extent, is an expression of singularity. In order to be judged as beautiful, indeed, singularity must also come with a perception of harmoniousness and, therefore, desirability, as though the aesthetic judgment were positively sanctioning those singular conjunctures that, albeit unrepeatable, seem to emerge from a sort of benign design, from the exertion of a kindly-minded abstract agency. Put more simply, when we come across a beautiful face, we do not likely see a young or old or even not a male or female face, but predominantly a beautiful face, that is, a face that is endowed with the mystery of an appealing singularity. The singularity in a face, therefore, cannot be the sign of anything when I first come across it. Upon such first encounter, the mind constructs a mental simulacrum of such a face, which coexists in the same time and in the same space of what has triggered the creation of such a mental simulacrum in the first instance, that is, the face itself.

At least since Kant, it is well known that the face must be perceived through such simulacrum, whose nature of simulacrum is actually revealed especially when the object that has led to its creation is manifestly not present anymore and, as a result, one is left with a mental simulacrum that is not in praesentia but in absentia. Through comparison between the two kinds of simulacra, then, one realizes that they share many features and possibly even the same nature. Thus, the experience of seeing a beautiful face for the first time is, to a certain extent, also an experience of recognition, not in the sense that such a face had been seen beforehand and that, as a consequence, the mind was already storing a mental simulacrum of the experience and awareness of having already seen the face, but in the much subtler sense that, while I am seeing a face for the first time - although this face might be entirely singular and, therefore, new to me - I must simultaneously construct a mental simulacrum of it that allows me to have also the experience of the awareness of seeing it. I see the face and at the same time I construct the ongoing memory of my being seeing it, which immediately turns into the awareness of such experience and will subsequently turn into the memory of such experience and its awareness.

From this point of view, any experience of singularity is a déjà vu, for it must entail the construction of a mental simulacrum of something in order to enable the experience of it and the awareness of the experience of it. A beautiful face seen for the first time is, from this point of view, always a remembered face, which we remember in the same moment as we see it for the first time, exactly by virtue of its singularity. Common, banal faces, which for some reason do not strike us for their uniqueness, remind us of other faces. To be reminded of something is the typical cognitive and mnemonic activity related to the token/type dialectic: a banal face reminds me of many other similar faces that I might have seen beforehand. A beautiful face, though, does not remind me of any other previously seen and memorized faces but is remembered as a singular face while it is being seen. As a consequence, although one might have the impression that a déjà vu is related to the circumstance of recognizing that which one has not experienced yet, it is actually related to the fact that experiencing something as a singularity, that is, experiencing something for the first time, entails the ongoing and simultaneous construction of the mental conditions for the possibility of its recognition. While I am admiring a beautiful face, I am simultaneously constructing the mental conditions that are allowing me to have an experience of it and the awareness of the experience of it, and that will actually allow me to remember it.

A déjà vu, then, is not abnormal in the sense that it completely subverts the laws of ontological experience and phenomenological cognition, but in the sense that it reveals, through a temporary malfunctioning of cognition, the laws by which it is constructed. In a déjà vu, the cognitive activity required to construct the mental simulacrum that allows one to have the experience and the awareness of the experience of something is, for some reason, temporally late in relation to the cognitive activity that is required to construct the mental simulacrum that allows one to have the memory of having had the experience and the awareness of the experience of something. Such a condition indicates that even that which is usually felt as the normal course of cognition, that is, seeing something, perceiving it, experiencing it, having the present awareness of such experience, and, subsequently, remembering both the perception, the experience, and the awareness, depends on the cooperation of several cognitive agencies, related to different areas of the neurophysiology of the brain, agencies whose correct synergy, indeed, produces an impression of unity. 
Slight misfunctioning of this synergy, though, immediately reveals the complex multiplicity that underpins any cognitive feeling of experience, awareness, and memory: I can see something because I am somehow also simultaneously constructing the memory of it, and it is only thanks to the illusion of awareness that I have the impression of seeing something for the first time before actually being in the condition of remembering it. That does not mean that the ontology of time is illusory, as countless mystical or paranormal interpretations of the déjà vu untenably surmise; that means, on the contrary, that the phenomenology of time is illusory, for it rests on a dynamic that cannot be simply chronological but that must be, on the opposite, semiotic. The ontology of perception is binding in the sense that I cannot construct the mental simulacrum of something if my senses have not first come into contact with it. From the phenomenological point of view, however, the mental simulacrum that allows me to experience something through the senses for the first time, the mental simulacrum that allows me to be aware of the fact that I am having this experience, and the mental simulacrum that either allows me to remember from the past such experience and its awareness or to imagine that I shall have this experience and its awareness again in the future, all these simulacra, then, share the same qualitative nature, which is essentially a semiotic one.

\section{The signification of singularity}

A singularity experienced for the first time is not a sign, although it might be turned into one by contrast and comparison with previously constructed mental models, patterns, and articulations. What is unique in a face, however, is not immediately perceived as a sign but as semiotic potentiality that gives rise to an actual semiotic experience only when the mental simulacrum stemming from perception is stretched into the mental simulacrum of awareness: it is in awareness, indeed, that a singular object is not only seen, but recognized as something that one is seeing and, therefore, as sign of itself in its own presence. There is a common misunderstanding in semiotics, according to which, since the most central semiotic experience is usually that which is had when mental simulacra refer to objects that are no longer co-present in time and space with the senses that have once experienced the object that these mental simulacra stand for, semiotic experience cannot be had in conditions of temporal and spatial co-presence. That would actually deny or at least neglect the simulacral nature of the mental constructs that are needed in order to experience something and be aware of such experience. When I see something, I do not experience it directly, but through a mental simulacrum of it whose construction, moreover, is influenced by a whole series of concomitant factors, including the linguistic and cultural articulation of reality that I have internalized as second nature and the particular conditions in which the other cognitive faculties that are involved in the holistic experience of perception and awareness work. When I am hungry and look at a sandwich, I do not have the same perception of it as I would when I am no longer famished.

A face that I am seeing, then, is not only a sign of other faces or of the idea or category of the face itself but also a sign of itself, meaning that what I am seeing is not the face itself but, as Kant would already posit, a mental simulacrum that signifies the face. I can, then, see the portrait of such face, that is, a visual representation of it, entailing a certain degree of reality effect. As counterintuitive as it might seem, the difference between a mental perception of a face and the mental perception of a visual representation of a face is only a quantitative one, not a qualitative one. The reason for which we can distinguish between the mental experience that we have when we see a face, and the mental experience that we have when we see the visual representation of a face, is purely technical, meaning that it is only because of the imperfection of the visual representation that we can actually tell it apart from the mental representation.

The more technological progress allows one to construct visual representations of objects that possess the same phenomenological characteristics of their mental representations in praesentia, the more the difference and the possibility of distinguishing between the former and the latter decreases. Am I seeing a red tulip, or the digital, 3D representation of a red tulip? The reason one still talks of "trompe l'oeil" is technical, not phenomenological; the perfect trompe-l'oeil does not allow one to distinguish between a mental representation stemming from an ontological reality and a virtual representation stemming from a simulation, for the first cannot be referred to its underpinning ontology. We do not experience reality but mental representations of it, so that representations that look exactly like those of our mind will be considered as non-mental only by convention. 


\section{The necessity of imperfect memory}

On the one hand, the human memory is imperfect. That is often seen as a limit to personal and collective success. Throughout history, individual and social mnemotechnics have been devised to improve the capacity of exerting a precise agency on memory as creator and deposit of both personal and community representations. Memory is the agency that creates and stores representations not only of that which is past but also of that which is absent, that is, of that which is either remote in time, meaning that once had an ontological existence but does not any more, or remote in space, meaning that once the senses were in contact with it, but they are not anymore, although the object of this contact might still enjoy a state of ontological existence in the present. It is important that memory functions consistently. That means that it is important that, for instance, we are able to consistently remember the number of our hotel room when the waiter asks us for it at breakfast. Similarly, it is important that we remember the face of a colleague that we have already met before in order to demonstrate our recognition of her or him when we meet again. At the same time, that which is frequently neglected perhaps exactly because of the influence of the comparison between the human memory and the memory that is enabled by non-human actors and devices, and in particular by digital mnemonic devices - is the importance of the imperfection of memory.

Throughout the natural evolution of the human species, the fact of being endowed with the cognitive ability of intentionally and unintentionally conjuring up in one's mind mental representations of absent entities has proven adaptive. Even the simple dynamic of learning through trial and error, for instance, would be impossible without this capacity - that is, without the ability of storing mental simulacra of the previously experienced harmful connotations of certain objects or situations of the environment. Thus, living beings endowed with this capacity have certainly enjoyed a significant evolutionary advantage in comparison to those living beings that, on the contrary, could not remember anything or had a more limited range of mnemonic abilities.

At the same time, the imperfection of memory has also proven equally adaptive. A famous short story by Jorge L. Borges (1942), "Funes el memorioso" ("Funes the Memorious") already indicates one key reason for which a too perfect memory might give rise to catastrophic consequences: mental synthesis would be impossible without the ability of neglecting and forgetting details. But another reason for the adaptive character of imperfect memory resides in the necessity to keep the distinction between mental simulacra of a present ontology and mental simulacra of an absent ontology, remote in time or in space. We can certainly close our eyes and conjure up in our mind the face of a departed lover. Even without closing the eyes and, therefore, without eliminating the influence of mental simulacra related to present perceptions of the senses, we can still make a mental effort and conjure up the image of such face. In no case, however, we shall be able to experience an awareness of the perception of this face as though it was that which we would experience when the departed lover was still physically in front of us. As much as this experience of the imperfection of memory might be unpleasant and even painful in many circumstances - for we would like to be able to conjure the face of the departed lover exactly as it was in flesh and bones - the semiotic discrepancy between the quality of mental simulacra that embody present perceptions and that of mental simulacra that embody absent perceptions is central to individual and social life as we know it.

\section{Hallucinating}

If the cognitive malfunctioning giving rise to déjà vus indicates what would happen if the synergy between the mental representation of a present perception and the mental representation of the awareness of a present perception overlapped, basically giving rise to the blurring of the awareness of the present in relation to the awareness of the future, another cognitive malfunctioning is equally revealing of the deep semiotic mechanisms of perception - that is, hallucinations. ${ }^{1}$ In hallucinations, and especially in retrospective hallucinations, the force by which mental simulacra of entities experienced before or elsewhere present themselves to the mind is such that they cannot be distinguished, in the theater of awareness, from mental representations of present ontological states. When memory works normally, I conjure up in my mind a pale mental simulacrum of a dead person, a simulacrum that progressively fades away as my present perception keeps remaining unexposed to the person; in hallucinatory memory, the mental simulacrum of what is not anymore, or of what still is but elsewhere, presents itself as so vivid as to make it impossible to distinguish between what I perceive and what I imagine. The imperfection of memory is fundamental to ensure the possibility of this distinction. 


\section{Towards the pan-mnemonicon}

In déjà vus, I have the impression that future ontological states enter my present; in hallucinations, I have the impression that past ontological states enter my present. Cultural semiotics should focus on the emergence of déjà vus and hallucinations not only in individual cognition but also in the temporal ideologies that characterize a semiosphere. ${ }^{2}$ It should, therefore, reflect on the impact of the adoption of mnemonic devices that challenge the principle of the imperfection of memory. Especially since the development of digital mnemotechnics, the ability to save it all, remember it all, and retrieve it all has been aggressively marketed and turned into a major source of profit. The reasons underlying this marketability of perfect memory are anthropological and even biological: a powerful memory is, indeed, a benefit for many species and individuals that are comparatively more endowed with it than others. Yet, the necessity of forgetfulness and oblivion is often neglected. The late Umberto Eco dwelled on the human quest for an art of forgetfulness ("ars oblivionalis" in Latin) (Eco and Migiel 1988). He concluded that, despite the efforts of several imaginative scholars across centuries, languages, and cultures, such art is impossible. Human beings can exert a certain agency on memory but not on forgetfulness. We remember both intentionally and unintentionally, but we forget only unintentionally. We cannot control oblivion, for this incapacity has probably turned into a capacity throughout evolution. Indeed, unwillingly remembering unpleasant memories might have protected us, and might still protect us, from incurring in the same harmful experiences over and over again.

Yet, memory, both the individual and the collective one, is not only nature and biology, it is also culture and society. The importance that we attribute to memory and remembering, the style by which we remember, and even the relation between remembrance and oblivion, are deeply influenced by a socio-cultural context. Technology is part of it, and especially technology that, by producing inscriptions and therefore 'documentality,' turns into mnemonic device (Ferraris 2009). Writing was a mnemonic device whose possibly deleterious effects on the mind and memory were already emphasized by Plato. With the digitalization of memory, new metaphors and ideals of memorization spread throughout the semiosphere. Digital memory is called "memory" only metaphorically, for it evidently differs from the human memory under many respects. First, it is a memory on which total control can be exerted, not only in writing but also in erasing traces. Second, it is presented and conceived as an unlimited repository. Third, unlike previous devices of memorization, it is insensitive to the signifiers of the signs whose signified it memorizes, for it can convert both visual and acoustic inputs into digits. Fourth, it ignores gustative, olfactory, and, to a very large extent, haptic sensorial manifestations. Fifth, it shows no real casualness and, therefore, no true serendipity, meaning that it retrieves memories of the past but does so only upon request. Attempts at simulating in digital machines the spontaneous resurgence of memories in human remembrance are still unsatisfactory. Similarly, the way in which digital devices seek to guide and assist but also influence the human memory is equally unspontaneous and unsurprising. In Facebook, for instance, an algorithm periodically proposes to us images from the past of our Facebook timeline, yet this randomness is mechanical and entirely controllable: if I want, I can tell Facebook not to remind me anymore of a lost lover; unfortunately (or, perhaps, fortunately), I cannot give the same instructions to my own memory. Sixth, the human memory is an embodied one, hence the imperfection and uncontrollability of its functioning.

Although digital memory is only metaphorically such, it still exerts a deep influence on the way in which the human memory is re-contextualized, conceived, and even trained and transformed. The fact that the importance of individual and also the collective oblivion is neglected, for instance, is undoubtedly a result of the increasingly widespread attitude of seeing the human memory as an inscribing surface that should ideally work like that of a digital device. The memory of machines is devised so as to empower the human memory and amplify some of its capacities, yet the peculiarities of the former, those of the digital memory, become in their turn a cultural pattern after which the latter and even the collective memory of a society are modeled.

As it was proposed earlier, the aesthetic encounter with singularity is possible thanks to the imperfection of memory as well as thanks to the jamming of the token/type mechanism. We have the impression of recognizing the source of a momentous aesthetic experience, such as a beautiful face, for instance, for this face challenges, exactly by virtue of its singularity, any existing categorization and typology: we must at the same time construct the mental simulacrum of the perception of such face and the mental simulacrum that, by stretching this simulacrum through the dimension of time, turns it into a signifier of itself, into a source of new, unprecedented meaning. The token/type mechanism is fundamental for the recognition of signifying patterns - yet, if its force was total, then no new meaning could arise since reality would be constantly deciphered as an occurrence of preexisting models or types. This, however, is the way in which most digital memory works, including that which enables the recognition of faces (through Eigenfaces dynamics, for instance). Digital memory offers a tremendous contribution to any present-day human activity. Our life without the memory of computers and similar devices would be intolerable in most circumstances. Yet, negligence or even oblivion of the essential differences between human and non-human memories and, even worse, the tendency to conceive, idealize, and 
market digital memory as the perfect model for any process of remembrance, to the point of devising a sort of 'pan-mnemonicon' - the mnemonic equivalent of the eighteenth-century panopticon - risks to turn cultural memory into a place for déjà vus and hallucinations.

\section{Conclusion: Déjà vus and hallucinations in collective memory}

Misfunctioning of the synergy between, on the one hand, the cognitive agency that constructs mental simulacra of the perception of reality and of the ongoing experience and awareness of it and, on the other hand, the cognitive agency that constructs mental simulacra through which a memory of past or distant perception, experience, and awareness of reality is stored leads, as it was pointed out before, to the somehow disquieting phenomenon of the déjà vu. As it was underlined supra, the feeling of recognition that is triggered by a déjà $\mathrm{vu}$ is apparently similar to the sensation of awareness that comes together with an intense aesthetic epiphany, such as, again, the first visual encounter with a beautiful face.

The similarity with the cognitive dynamic of a déjà vu is actually only apparent: true, in a déjà vu too the synergy between perception, apperception, awareness, and memory is altered so as to bring about an overlapping of the feeling of the past, that of the present, and that of the future; in a déjà vu, however, an experience that is in effect singular - for instance, a sentence uttered for the first time - is made sense of through an abnormal application of the type/token dialectics, which even contrasts the logic of time and categorizes the singularity of the present instant as 'already experienced.' In the intense aesthetic encounter with singularity, on the contrary, such as the experience of beauty, for example, exactly the opposite happens: the singular is not categorized through preexisting models but gives rise to a new structuration of the entire field of meaning. After we come across an extremely beautiful face for the first time, the meaning of what a face is, and especially the meaning of what a face is for us, must be entirely rewritten.

That explains also the completely diverging emotional tone that underlies, on the one hand, déjà vus, and, on the other hand, aesthetic epiphanies. The former are disquieting for they are sensed as a meaningless overflowing of the future into the present: what is for the first time is categorized as a returning future. The latter are, on the contrary, euphoric because they are experienced as a meaningful overflowing of the present into the future: what is for the first time is categorized as blossoming being.

In a similar way, hallucinations arise because what should be uniquely perceived in a different time, that is, the past, and in a different space (a remote space) comes into contact with perception with abnormal evidence. In this case too, what should be a singularity in the past 'invades' the present, leaving one with the puzzling sensation of not being able to distinguish between the mental simulacrum of a past memory and that of a present perception. Whereas a déjà vu is meaningless overflowing of the future into the present, hallucinations are meaningless overflowing of the past (or the remote) into the present.

"Collective memory" is not simply a metaphor. The emergence of shared memory through the construction of artifacts of various kinds encouraging the members of a community to retain similar mental simulacra of the past works in a way that significantly differs from that of the construction of individual memory. We are not always sure of our individual memories yet the degree of certainty that we tend to attribute to the recollection of personal experience is certainly much higher than that which we are ready to grant to collective memory. The phenomenological and semiotic distinction between individual and community memory would deserve a special consideration, together with the multiple relations of construction and deconstruction between the two. On the one hand, it is reasonable to believe that the dialectics between micro-interactions and macroconfigurations that characterize the exchange between "langue" and "parole" in the structural understanding of language evolution might also play a significant role in the formation of collective memory. This too, indeed, might stem as largely unintentional macro-effect of personal memories. Moreover, attempts at institutionalizing memories might exert a significant influence (although with a different degree of efficacy) than, for instance, attempts at institutionalizing languages.

One should not neglect, however, that memory is a multi-modal domain in which the institution of intersubjective trends, regularities, and patterns is necessarily less compelling than that which characterizes the structuration brought about by that which Jurij M. Lotman would call the "primary modeling system" of semiospheres, that is, verbal language. The morphology of a verbal language is usually highly and strictly codified. Its semantics are not, yet we might reasonably believe that if we utter the word "dog," then our interlocutor will refer this signifier to a signified that is similar to that which we have in mind when we utter such word. But will our interlocutor conjure in her or his mind the same memories of actual dogs that emerge in our memory when we think about dogs? That is most probably not the case. Personal memories are fleeting collections of signs of different kinds, which nevertheless entirely remain within the semantic sphere. Societies can institutionalize mementos but cannot institutionalize memories. They can reach a stable agreement so as to monumentalize the 
memory of a past event, considered as significant for the whole community, but cannot enforce the meaning of monuments on individual memories. ${ }^{3}$ That is, for instance, at the semiotic source of the excruciating problem of handing down to future generations the memory of a tragic historical event like the Shoah: many European cities now contain several memorials of the tragedy, yet these memorials are useless if they do not manage to stir personal memories into the formation of a long-lasting shared remembrance. European cities are also full of statues representing historical characters that once deserved to be represented in this guise but whose name and, above all, deeds are now completely absent from the present-day shared memory of the urban collectivity.

Despite the evident discrepancy in the functioning of subjective memory on the one hand and intersubjective memory on the other, the relation between the two is complex but not entirely metaphoric. Societies do remember and share community simulacra of the past that acquire an autonomous status and intertwine with personal representations of bygone experiences. That is the reason for which the observation of abnormal mnemonic processes in individual memories can be extended to societies as well, mutatis mutandis of course. The collective memories of societies too can produce déjà vus and hallucinations. More precisely, these cognitive fibrillations can inflect social trends of collective memory construction. As it was pointed out earlier, the massive introduction of digital memories and their idealization as the utopian model for human and embodied memory is one of the main causes for the appearance of these fibrillations, although always in conjunction with the many other factors that mold the temporal, aspectual, and mnemonic ideologies of a society.

In societies that uncritically embrace and endorse a digitalization not only of memory but of the same idea of memory, déjà vus manifest themselves as the impossibility of subtracting culture from a disquieting feeling of recognition. For instance, the quantity and speed of entertainment production increase steadily, yet consumers cease to have the experience of singularity, for the type/token dynamic that underpins large-scale cultural infrastructures and production leaves no residues for the creation of new potentialities of significance and meaning: a new TV series is inevitably received as nothing but the Nth occurrence of a format that is so constraining as to exert a decisive impact on the reception of semantic contents. In déjà vu cultures, individuals more and more no longer come across texts but instances of genres that repeat themselves with mechanic variations, exuding an aesthetic value that is merely combinatorial, a matter of style. This collective feeling of 'already done, already seen' tragically starts to involve perceptions, experiences, and simulacra that long term anthropological valorizations qualify as singular or unique, like the death of a human being, for instance. Déjà vu digital memories are unable to attach an aesthetic epiphany to the large-scale representation of death, and even of violent death, for the exclusivity of the ontology of tragedy is absorbed by the format of the simulacrum: poetry is the real antidote to the digital decline of singularity, yet few widely circulating texts can, in a present-day digital society, promote a poetic reworking of collective memory in the sense of singularity. Most post-modern societies relegate the poetic discourse (including that of the other arts) at the elitist margins of the semiosphere.

As regards hallucinations, which in relation to individual cognition have been defined as the abnormal encroaching of mental simulacra of an imagined past into the present perception, apperception, and awareness, they too can affect semiospheres as well, inflecting the therein predominant temporal and aspectual ideologies. A hallucinating culture is one that is no longer able to distinguish between its fears and its current state. It is a culture in which fake news proliferates because individuals have lost contact with the capacity for distinguishing what is present to perception from what is presentified to it. If poetry is the only remedy for a semiosphere suffering from abnormal déjà vus, the only antidote against hallucinations is philology, meant as the disciplined capacity to articulate the difference between simulacra with an ontological dignity and mere figments of the imagination.

We should start cultivating our poetry. We should start perfecting our philology. We should adapt both to the infrastructure of the digital world so as to discover a new sense of singularity and a new sense of reality, lest déjà vus and hallucinations invade more and more our shared memory.

\section{Notes}

1 Literature on hallucinations is also extensive. See West (1962); Siegel and West (1975); Aleman and Larøi (2008, 2010); Marshall (2000); Blom and Sommer (2012); Sacks (2012) and Jardri et al. (2013). In semiotics, Peirce's reflection on dreams often involves a philosophy of the meaning of hallucinations; on this aspect, see West (2016); for a semiotics of hallucinations in schizophrenia, Flexer (2016). Classical phenomenological accounts of hallucinations that are akin to those of semiotics are also in Husserl (Ideas II) and, of course, in MerleauPonty's Phenomenology of Perception.

2 In Lotman's semiotics, the semiosphere is the conceptual space in which cultures pattern the social circulation of meaning.

3 Much of Umberto Eco's semiotics revolves around the study of the cultural regularities that bring about a community of interpreters. This ultimately consists in negative more than in positive terms, that is, in a perimeter within which the game of interpretation can be freely played, either individually or in groups, but beyond which one falls into unreasonable and even abusive textual exegesis; see Eco 1987. The limits of interpretation, however, are much more porous as regards intimate memories, whose referent does not necessarily leave an inter-subjective trace in the cultural environment. Members of a same family, for instance, may hold completely different versions of a 
past event in their respective memories, if these have not been somehow constrained by the fixation of a system of signs into an external and, therefore at least to a certain extent, inter-subjective souvenir.

\section{References}

Aleman, A. \& F. Larøi. 2008. Hallucinations: The science of idiosyncratic perception. Washington: American Psychological Association. Aleman, A. \& F. Larøi (eds.). 2010. Hallucinations: A guide to treatment and management. Oxford and New York: Oxford University Press. Anderson, D. 1984. Peirce on metaphor. Transactions of the Charles S. Peirce Society 20(4). 453-468.

Blair, R. D. G. 2012. Temporal lobe epilepsy semiology. Epilepsy Research and Treatment 2012. Article ID: 751510. www.hindawi.com/journals/ert/2012/751510/cta/ (accessed 10 March 2019).

Blom, J. D. \& I. E. C. Somme (eds.). 2012. Hallucinations: Research and practice. New York: Springer.

Bodei, R. 2006. Piramidi di tempo: teoria e storie del déjà vu. Bologna: Il mulino.

Brown, A. S. 2004. The Déjà Vu experience. New York: Psychology Press.

Bruneau, T. 2012. Chronemics: Time-binding and the construction of personal time. ETC: A Review of Ceneral Semantics 69(1). 72-92.

Eco, U. \& M. Migiel. 1988. An Ars Oblivionalis? Forget It! PMLA 103(3). 254-261.

Ferraris, M. 2009. Documentalità: Perchéè necessario lasciar tracce. Rome and Bari: Laterza.

Flexer, M. J. 2016. The Schizophrenic Sign: A Dialectic of Semiotics and Schizophrenia. Leeds: University of Leeds PhD thesis.

Houppermans, S., J. Jacobs \& R. Kruk (eds.). 2011. Déjà vu: Herhaling in literatuur en kunst. Leiden: Leiden University Press.

Jansen, J. 1991. Das Déjà vu-Erlebnis. Frankfurt am Main and New York: P. Lang.

Jardri, R., et al. (eds.). 2013. The neuroscience of hallucinations. New York: Springer.

Jones, M. D. \& L. Flaxman (eds.). 2019. The Déjà Vu Enigma: A Journey through the anomalies of mind, memory, and time. Franklin Lakes: New Page Books.

Krapp, P. 2004. Déjà Vu: Aberrations of cultural memory. Minneapolis: University of Minnesota Press.

Marshall, J. C. 2000. The semiotics of schizophrenia: Unica Zürn's artistry and illness. Modern Language Studies 30(2). 21-31.

Pozzato, M. P. 2017. Retentissement, résonance e déjà vu: Attorno a Benveniste e agli effetti di soggettività nei testi. E/C. http://www.ec-aiss.it/ (accessed 10 March 2019).

Sacks, O. 2012. Hallucinations. New York: Alfred A. Knopf.

Siegel, R. K. \& L. J. West (eds.). 1975. Hallucinations: Behavior, experience, and theory. New York: Wiley.

Sno, H. N. 1993. The Déjà Vu Experience: A Psychiatric Perspective. Amsterdam: University of Amsterdam PhD thesis.

Strongman, L. 2012. Déjà vu explained? A qualitative perspective. The Journal of Mind and Behavior 33(3/4). $205-218$.

West, D. 2016. Peirce's creative hallucinations in the ontogeny of abductive reasoning. Public Journal of Semiotics 7(2). 51-71.

West, L. J. (ed.). 1962. Hallucinations. APA-AAAS Symposium on Hallucinations. New York: Grune and Stratton.

Zantides, E. 2016. Visual metaphors in communication: Intertextual semiosis and déjà vu in print advertising. Romanian Journal ofCommunication and Public Relations 18(3). 65-74.

\section{Bionotes}

Massimo Leone is a full tenured professor ("professore ordinario") of cultural semiotics, visual semiotics, and philosophy of communication at the Department of Philosophy and Educational Sciences, University of Turin, Italy as well as a permanent part-time visiting full professor of semiotics in the Department of Chinese Language and Literature, University of Shanghai, China. He is a 2018 ERC Consolidator Grant recipient, the most important and competitive research grant in Europe. 